\title{
The Corylus mandshurica genome provides insights into the evolution of Betulaceae genomes and hazelnut breeding
}

\author{
Ying $\mathrm{Li}^{1}$, Pengchuan Sun $\mathbb{D}^{2}$, Zhiqiang $\mathrm{Lu}^{3,4}$, Jinyuan Chen ${ }^{1}$, Zhenyue Wang ${ }^{1}$, Xin Du' ${ }^{1}$ Zeyu Zheng ${ }^{1}$, Ying $\mathrm{Wu}^{1}$, \\ Hongyin $\mathrm{Hu}^{1}$, Jiao Yang ${ }^{1}$, Jianxiang Ma', Jianquan Liu, ${ }^{1,2}$ and Yongzhi Yang
}

\begin{abstract}
Hazelnut is popular for its flavor, and it has also been suggested that hazelnut is beneficial to cardiovascular health because it is rich in oleic acid. Here, we report the first high-quality chromosome-scale genome for the hazelnut species Corylus mandshurica $(2 n=22)$, which has a high concentration of oleic acid in its nuts. The assembled genome is $367.67 \mathrm{Mb}$ in length, and the contig N50 is $14.85 \mathrm{Mb}$. All contigs were assembled into 11 chromosomes, and 28,409 protein-coding genes were annotated. We reconstructed the evolutionary trajectories of the genomes of Betulaceae species and revealed that the 11 chromosomes of the hazelnut genus were derived from the most ancestral karyotype in Betula pendula, which has 14 protochromosomes, by inferring homology among five Betulaceae genomes. We identified 96 candidate genes involved in oleic acid biosynthesis, and 10 showed rapid evolution or positive selection. These findings will help us to understand the mechanisms of lipid synthesis and storage in hazelnuts. Several gene families related to salicylic acid metabolism and stress responses experienced rapid expansion in this hazelnut species, which may have increased its stress tolerance. The reference genome presented here constitutes a valuable resource for molecular breeding and genetic improvement of the important agronomic properties of hazelnut.
\end{abstract}

\section{Introduction}

The hazelnut genus Corylus L., which belongs to the family Betulaceae, contains $\sim 20$ species, all bearing edible nuts $^{1,2}$. Hazelnuts, which are a favorite among consumers, are widely used in food processing and in the manufacture of confectionery products, including chocolate, biscuits, and hazelnut oil. Hazelnuts have a high overall content of fatty acids ( $60 \%$ of the hazelnut kernel), mostly oleic acid ( $80 \%$ of the fatty acids), which are important dietary components for humans ${ }^{3-5}$. Oleic acid is usually regarded as a healthy fatty acid, as it can reduce the risk of

\footnotetext{
Correspondence: Yongzhi Yang (yangyongzhi2008@gmail.com)

'State Key Laboratory of Grassland Agro-Ecosystem, Institute of Innovation Ecology \& School of Life Sciences, Lanzhou University, Lanzhou, China

${ }^{2}$ Key Laboratory of Bio-Resource and Eco-Environment of Ministry of Education \& State Key Laboratory of Hydraulics \& Mountain River Engineering, College of Life Sciences, Sichuan University, Chengdu, China

Full list of author information is available at the end of the article

These authors contributed equally: Ying Li, Pengchuan Sun.
}

cardiovascular disease by inhibiting cholesterogenesis in vivo as well as in vitro, reducing blood pressure, and inhibiting the atherosclerotic process ${ }^{6-8}$. Currently, the major hazelnut-producing countries are Turkey, Italy, Spain, and the USA, and the total annual global hazelnut production is in excess of 550,000 tons (http://www.fao. org/3/x4484e/x4484e03.htm).

Hazelnut has a long history of utilization and production, likely predating the Roman era ${ }^{9}$, and the most widely cultivated species have been European hazelnut (Corylus avellana), which has been bred mainly for high fruit yield. European hazelnut is usually susceptible to diseases such as eastern filbert blight (EFB), which can cause serious damage to the commercial production of European hazelnut ${ }^{10}$. During the long history of hazelnut usage, people have focused on increasing kernel size, oil content, and disease resistance $^{11,12}$. With the development of molecular plant breeding, an increasing number of technologies are being

\section{() The Author(s) 2021}

(c) (i) Open Access This article is licensed under a Creative Commons Attribution 4.0 International License, which permits use, sharing, adaptation, distribution and reproduction c. in any medium or format, as long as you give appropriate credit to the original author(s) and the source, provide a link to the Creative Commons license, and indicate if changes were made. The images or other third party material in this article are included in the article's Creative Commons license, unless indicated otherwise in a credit line to the material. If material is not included in the article's Creative Commons license and your intended use is not permitted by statutory regulation or exceeds the permitted use, you will need to obtain permission directly from the copyright holder. To view a copy of this license, visit http://creativecommons.org/licenses/by/4.0/. 
utilized, especially CRISPR-Cas9 editing, which can greatly reduce the length of the breeding cycle and improve quality and efficiency ${ }^{13}$. Genes related to fatty acid biosynthesis and oleic acid accumulation are important targets for future breeding to improve the quality of the oil content. One recent study generated cotton with a high oleic acid content using the CRISPR/Cas9 system to knock out a microsomal $\omega-6$ fatty acid desaturase gene, the product of which can catalyze the desaturation of oleic acid to form linoleic $\operatorname{acid}^{14}$. In the case of disease resistance, the focus is usually on disease-resistance $(\mathrm{R})$ proteins, which help plants defend against a range of pathogenic organisms, including parasites, fungi, bacteria, oomycetes, insects, and viruses ${ }^{15}$. The largest class of disease-resistance $\mathrm{R}$ genes, those encoding nucleotide-binding site (NBS) proteins, have a critical role in defending plants from a multitude of pathogens and pests ${ }^{16}$. However, identifying such important gene resources in hazelnut has been difficult because of the lack of a highquality genome assembly.

In China, large-scale cultivation of hazelnut is rare, but wild varieties, mainly $C$. heterophylla Fisch. and C. mandshurica Maxim., are distributed in mountainous forest belts and deep valleys at high altitudes in northern and northeastern China ${ }^{1}$. C. mandshurica is a deciduous shrubby Asian hazel, the bracts of which form a tubular husk with pubescence and setae; it grows naturally or is artificially cultivated across much of eastern and northern Asia. The nuts of C. mandshurica are characterized by a thin husk, a high kernel weight to hazelnut weight ratio, and a high concentration of oleic acid ${ }^{3}$. C. mandshurica is also highly resistant to $\mathrm{EFB}^{17}$, and interspecific cross-breeding trials have been carried out between C. mandshurica and European hazelnut to utilize the former's valuable agronomic properties ${ }^{18}$. Thus, a C. mandshurica genome is urgently needed for use in hazel breeding.

In addition to the need to identify key gene resources in hazel, elucidating the evolutionary history of the genome across the Betulaceae is also important to help us understand the evolution of specific traits. Two subfamilies are recognized in the Betulaceae: Alnus $(2 n=28)$ and Betula $(2 n=28)$ form the Betuloideae, and the other four genera comprise the Coryloideae. Within the Coryloideae, Corylus $(2 n=22)$ and Ostryopsis $(2 n=16)$ are successively sister to Carpinus $(2 n=16)$ and Ostrya $(2 n=16)^{1,19,20}$. Although the Betuloideae diverged early as a monophyletic lineage, the hazelnut genus Corylus seems to possess many traits similar to those of the Betuloideae ${ }^{21}$. All of these traits indicate that the evolutionary origins of the hazelnut genus, especially chromosomal evolution, in the Betulaceae need further examination.

Although a rough genome for European hazelnut $(C$. avellana) based on Illumina sequencing has been released ${ }^{22}$ (https://hazelnut.data.mocklerlab.org/), the biosynthesis of oleic acid and the types of NBS in hazelnuts remain unclear due to the low quality of the assembled genome and poor annotation of the relevant genes. In this study, we constructed a high-quality chromosome-level reference genome for C. mandshurica by combining Illumina short reads, Nanopore long reads and chromosomal conformational capture (Hi-C) sequencing reads. The high-quality genome for a hazelnut species presented here helps us to clarify the biosynthesis of oleic acid in hazelnuts, investigate disease resistance in this species and infer the evolutionary origin of the hazelnut genus. This genomic resource also provides a valuable foundation for improving our agronomic understanding of hazelnut and carrying out molecular breeding in the future, including cloning key genes that control hazelnut traits and identifying disease-resistance alleles.

\section{Results}

\section{Genome sequence and assembly}

A mature C. mandshurica tree growing in Xinglong Mountain National Nature Reserve was selected for de novo whole-genome sequencing (Fig. 1). A total of 38.78 Gb ( 98.90×, Supplementary Table 1) of Illumina clean short reads was retrieved and used to perform a genome survey, which indicated that the size of the $C$. mandshurica genome was $392.16 \mathrm{Mb}$ and that it has relatively high heterozygosity $(\sim 0.92 \%)$ (Supplementary Fig. 1). The estimated genome size is very close to that previously reported for European hazelnut ${ }^{22}(378 \mathrm{Mb})$. A total of $38.08 \mathrm{~Gb}(\sim 97.12 \times$, Supplementary Table 1$)$ of raw Nanopore long reads were self-corrected, assembled, and polished with NextDenovo and Nextpolish ${ }^{23}$. After deletion of candidate allelic haplotigs, the final contig-level assembly of $367.67 \mathrm{Mb}$ was obtained with a contig N50 of $14.85 \mathrm{Mb}$ (accounting for $93.7 \%$ of the estimated genome size; Table 1 and Supplementary Table 2). To evaluate our assembly, first, Benchmarking Universal Single-Copy Orthologs $^{24}$ (BUSCO) was selected, and the results showed that $97.1 \%$ of conserved genes could be completely annotated within our genome, which is more than has been achieved for other published Betulaceae species $^{22,25-29}$ (Supplementary Table 3). The base accuracy of the assembly was also estimated based on the mapping of Illumina reads. We aligned genome sequencing reads and transcriptome sequencing reads to the $C$. mandshurica genome, with mapping rates of $98.86 \%$ and $99.68 \%$, respectively (Supplementary Table 4). The RNA transcripts were further aligned, and $97.00 \%-99.37 \%$ of transcripts could be mapped with over more than half their length to the same contig (Supplementary Table 5). These results collectively suggest that the $C$. mandshurica genome is well assembled and of high quality.

We also generated $42.04 \mathrm{~Gb}$ of clean reads from $\mathrm{Hi}-\mathrm{C}$ sequencing, giving $\sim 114.43 \times$ of the $C$. mandshurica genome (Supplementary Table 6). The quality of $\mathrm{Hi}-\mathrm{C}$ data was assessed by mapping the reads to the assembled genome, 

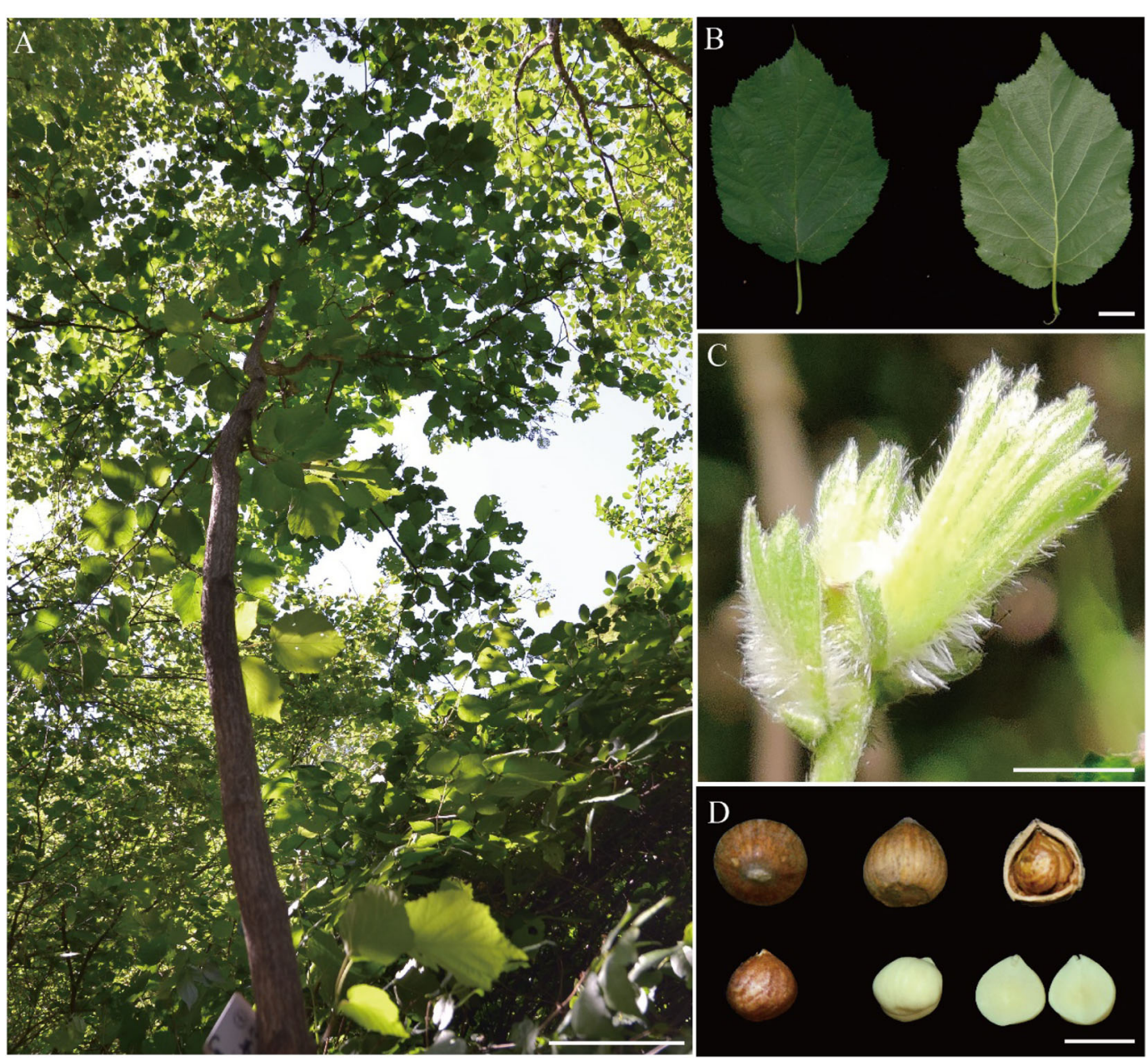

Fig. 1 Corylus mandshurica, leaves, tender fruiting bracts, and hazelnuts. A Photograph of a mature C. mandshurica tree $(\sim 4.8 \mathrm{~m})$ growing in Xinglong Mountain National Nature Reserve that was selected for de novo whole-genome sequencing. Scale bars $(0.5 \mathrm{~m})$ is shown at the bottom right. B Mature leaves of C. mandshurica, the front surface on the left, back on the right. C Cluster of tender fruiting bracts of $C$. mandshurica with obvious pubescence and setae. D Nuts of C. mandshurica. Top panel from left to right: top view, lateral view, and lateral view of nut in the shell. Bottom panel from left to right: lateral view of the nut with spermoderm, lateral view of the kernel, and cross-section of nut. Scale bars (1 cm) are shown at the bottom right in $\mathbf{B}-\mathbf{D}$

with $92.3 \%$ of reads being mapped to the assembled contigs, and the unique mapped read pair percentage was 50.5\% (Supplementary Table 6). Valid interaction pairs were integrated from the unique paired alignments, and all the contigs were categorized and ordered to construct chromosome-scale scaffolds, resulting in $11 \mathrm{C}$. mandshurica pseudomolecules totaling $367.67 \mathrm{Mb}$ with a scaffold N50 of $36.3 \mathrm{Mb}$ (Table 1, Fig. 2A, and Supplementary Fig. 2). The longest and shortest pseudomolecules were chromosomes 1 and 11 , with lengths of 52.98 and $21.94 \mathrm{Mb}$, respectively (Supplementary Table 7).

\section{Genome annotation}

The C. mandshurica genome was found to contain 252.7 Mb (68.74\%) transposable elements (TEs) (Fig. 2A and Table 1), specifically, 63.48\% retrotransposons and $4.39 \%$ DNA transposons (Supplementary Table 8 ). The dominant repetitive sequence type is long terminal retrotransposons (LTRs), forming $57.92 \%$ of the repetitive sequences, whereas $19.95 \mathrm{Mb}(5.46 \%)$ of the genome consists of non-LTR elements (Supplementary Table 8). A combination of transcriptomics, homology information, and a de novo approach were used to accurately predict gene models. We predicted 28,409 genes in the $C$. mandshurica genome (Fig. 2A and Supplementary Table 9). The average protein-coding gene size was $3562 \mathrm{bp}$, and the mean exon number was 5.0 (Table 1). The predicted protein-coding genes spanned a total length of $101.19 \mathrm{Mb}$ and were anchored to the 11 chromosomes (Fig. 2 and Supplementary Table 9). In addition, 1327 
Table 1 Statistical details of genome assembly and annotation

\begin{tabular}{ll}
\hline Assembly & Size (bp) \\
\hline Genome assembly & \\
Contig N50 & $14,849,403$ \\
Contig N90 & $3,939,978$ \\
Longest contig & $22,635,284$ \\
Total contig length & $367,672,720$ \\
BUSCO (complete) & $97.1 \%$ \\
Genome annotation & \\
No. of predicted protein-coding genes & 28,409 \\
Average gene length & 3561.82 \\
Mean length of exons per gene & 5.0 \\
Masked repeat sequence length & $252,743,315$ \\
Percentage of repeat sequence & $68.74 \%$ \\
Hi-C assembly & \\
Scaffold N50 & $36,270,330$ \\
Scaffold N90 & $22,830,127$ \\
Longest scaffold & $52,982,101$ \\
Total scaffold length & $367,672,720$ \\
\hline
\end{tabular}

(92.2\%) $\mathrm{BUSCO}^{24}$ genes could be completely matched to our predicted $C$. mandshurica gene set (Supplementary Table 10). Among the 28,409 genes predicted, 26,309 (92.6\%) were functionally annotated from five databases of known proteins: $\operatorname{TrEMBL}^{30}(83.3 \%)$, SWISS-PROT ${ }^{30}$ (66.4\%), Gene Ontology (GO, 56.0\%), Kyoto Encyclopedia of Genes and Genomes ${ }^{31}$ (KEGG, 28.4\%) and InterPro (92.6\%) (Supplementary Table 11). Additionally, 1366 transcription factors were detected in the C. mandshurica genome (Supplementary Table 12). We used transcription factors from $C$. avellana for further comparison. We found that four transcription factor families were significantly expanded $(p<0.01)$ in $C$. mandshurica compared to $C$. avellana. Three of these families were related to stress responses: APETALA 2/ ethylene response factor (AP2/ERF-ERF) ${ }^{32}$, calmodulinbinding transcription activator (CAMTA) ${ }^{33}$, and v-myb avian myeloblastosis viral oncogene homolog $(\mathrm{MYB})^{34}$. The basic/helix-loop-helix (bHLH) ${ }^{35}$ family, which is related to cell proliferation, was also significantly expanded in C. mandshurica (Supplementary Table 12). Noncoding RNAs were annotated, giving predictions of 195 transfer RNA (tRNA) genes, 336 ribosomal RNA (rRNA) genes, 329 small nuclear RNA genes, and 83 microRNA (miRNA) genes (Supplementary Table 13).

\section{Genome evolution}

To explore genome evolution in C. mandshurica, genes from 12 species in total, including five species of Betulaceae (Betula pendula ${ }^{25}$, C. avellana $^{22}$, Carpinus fangiana ${ }^{26}$, Ostrya rehderiana ${ }^{27}$, and Ostryopsis nobilis ${ }^{28}$ ), three species of other Fagales (Casuarina equisetifolia ${ }^{36}$, Juglans regia ${ }^{37}$, and Quercus robur ${ }^{38}$ ), Arabidopsis thaliana $^{39}$, Fragaria vesca ${ }^{40}$, and Vitis vinifera ${ }^{41}$, were clustered into 31,991 gene families (Supplementary Fig. 3). Out of these, we identified 1628 single-copy genes and reconstructed a phylogenetic tree with this gene set (Fig. 2C). This result revealed that C. mandshurica and $C$. avellana diverged from one another $\sim 11.25$ million years ago (Mya), placing them in the basal position in the Coryloideae, and diverged from the Coryloideae $\sim 21.15$ Mya. Betulaceae were estimated to have diverged from the other three Fagales species, casuarina, walnut, and oak, $\sim 51.03, \sim 65.08$, and $\sim 70.97$ Mya, respectively. The Ks distribution was determined using syntenic paralogs from each genome. The Ks value for collinear gene pairs indicated that there had been no recent whole-genome duplication in any of the six Betulaceae species, but the eudicot-common hexaploidy event was observed (Fig. 2B).

\section{Inference of karyotype evolution in Betulaceae}

Genomic changes can be detected and relative chronometry established by parsimony-based phylogenomic analysis, and evolutionary trajectories of karyotypes can also be elucidated in this way. Here, to reveal the karyotype evolutionary trajectories of Betulaceae, we identified syntenic conservation and chromosome rearrangements among the five genomes of $B$. pendula (Bpe), C. mandshurica (Cma), Ca. fangiana (Cfa), Ostrya rehderiana (Ore), and Ostryopsis nobilis (Ono) to represent the genera Betula, Corylus, Carpinus, Ostrya, and Ostryopsis, respectively (Fig. 3 and Supplementary Figs. 47). Putative homologous genes and collinear genes were identified within each genome and between each pair of genomes (Fig. 2D), and dot plots for species within Betulaceae were employed to depict orthology information (Supplementary Figs. 4 and 5).

B. pendula was identified as the most primitive species among these five Betulaceae based on previous studies, so the karyotype of $B$. pendula should be the closest to the ancestral karyotype ${ }^{21,42,43}$. We, therefore, selected $B$. pendula as the reference to identify syntenic blocks across these genomes. Analysis of syntenic relationships showed that the integrity of all chromosomes except Bpe1 and Bpe3 of B. pendula was essentially preserved in C. mandshurica, and seven chromosomes showed one-to-one correspondence between the two species (Supplementary Fig. 4). There were no corresponding chromosome pairs between $B$. pendula and Ca. fangiana, Ostrya rehderiana or Ostryopsis nobilis, but all of them showed clear evidence 


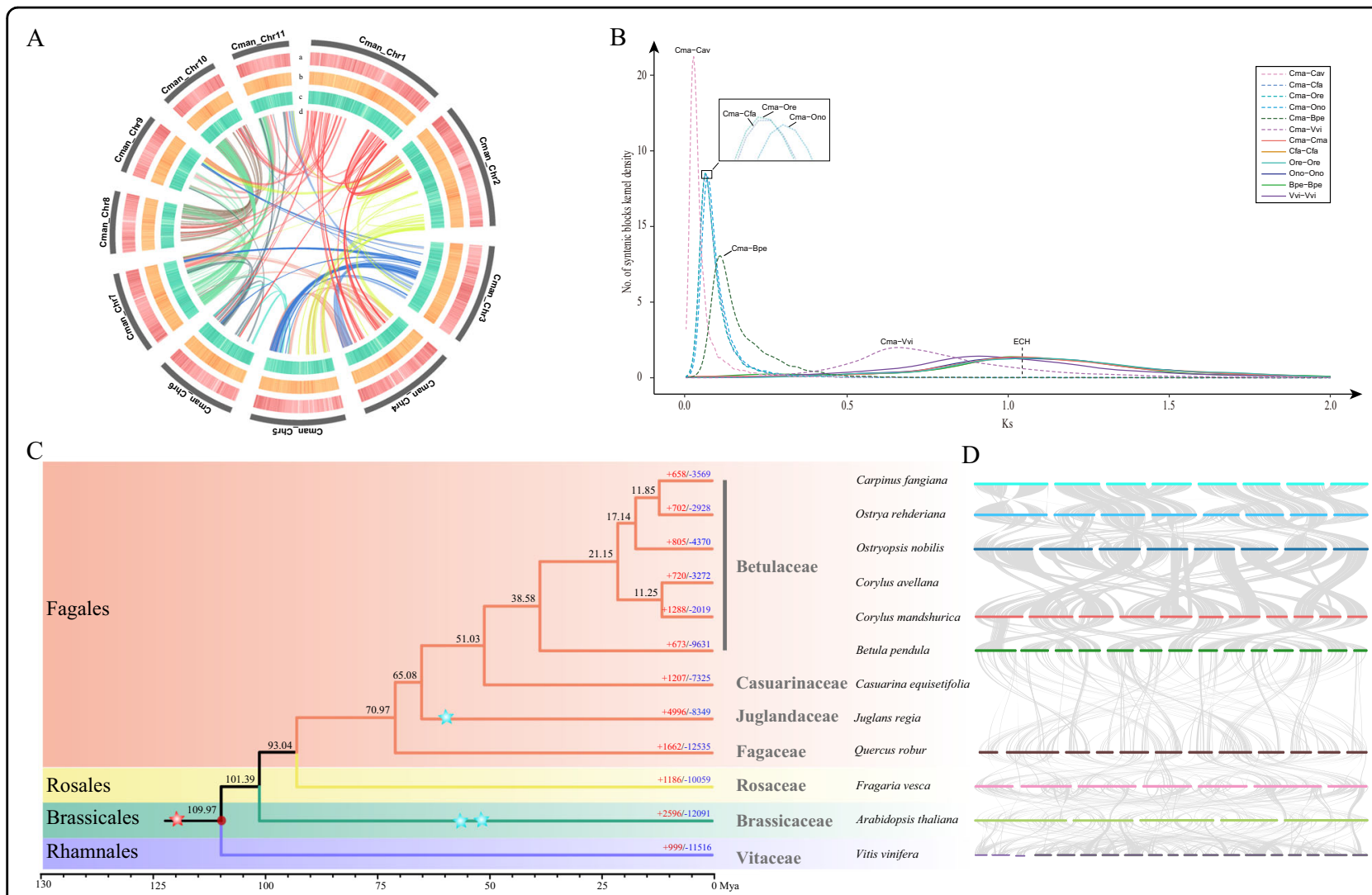

Fig. 2 Genome features and evolutionary and comparative genomic analyses. A Mapped features of the C. mandshurica genome, including a GC (guanine-cytosine) content, b repeat density, c gene density, d synteny information, Cma C. mandshurica. B Ks distribution of syntenic blocks between and within Betulaceae species. Bpe: B. pendula; Cav: C. avellana; Cfa: Ca. fangiana; Cma: C. mandshurica; Ono: Ostryopsis nobilis; Ore: Ostrya rehderiana; Vvi: Vitis vinifera; ECH: Eudicot-common hexaploidy. The enlarged display of the peak of the curve for the three Ks plots is shown in the black box. C Phylogenomic tree and expansion and contraction of gene families among C. mandshurica and 11 other species. The calibration time is marked by a red point. The blue stars and red stars represent the known whole-genome duplication and whole-genome triplication events identified previously, respectively. D MCscanX identified synteny blocks (involving $\geq 5$ collinear genes) between nine species containing chromosome-scale genomes. The species names correspond to those in $\mathbf{C}$

for retention of complete B. pendula chromosomes (Supplementary Figs. 5-7). Large regions of chromosome segments shared by extant genomes can be used to infer the identities of other protochromosomes. However, there were no shared chromosome segments even though several B. pendula chromosomes (chromosomes 1, 3, 4, 6, 11, 12 , and 14) appeared as rearrangements in all four other species (Fig. 3 and Supplementary Figs. 4-7), indicating that $B$. pendula chromosomes correspond to the ancestral karyotype of Betulaceae and have not undergone large chromosome segment rearrangements.

As explained above, we used B. pendula chromosomes as the ancestral karyotype to infer evolutionary trajectories for each Coryloideae species. The formation of C. mandshurica chromosome 1 (Cma1) could be clearly inferred as having occurred by a fusion of Bpe1 and Bpe3 and a nested chromosome fusion (NCF) of Bpe11 (Fig. 3B). The NCF process could have occurred as follows: Bpe1 and Bpe3 reciprocally translocated chromosome arms (RTA) and formed $\mathrm{Cma} 3$ and the precursor of Cma1; Cma3 consisted of part of the short arm of Bpe3 and most of Bpe1, while the precursor of Cmal consisted of part of the short arm of Bpe1 and the long arm of Bpe3; then Bpe11 underwent crossing over to form a major chromosome and a satellite chromosome; the satellite chromosome may have been lost, while the major chromosome was inserted into the Bpe3 long arm region of the precursor of Cma1. $\mathrm{Cma} 2$ was formed from Bpe4 and Bpe6 by end-end joining (EEJ) (Fig. 3C), and Cma4 was formed by an NCF of Bpe14 into Bpe12 (Fig. 3D).

During the process of construction of C. mandshurica chromosomes, two intrachromosome telomere-proximal crossings occurred to generate two free-end intermediate chromosomes and two satellite chromosomes (Fig. 3B-D); the former fused into the pericentromeric regions of other chromosomes, while the satellite chromosomes may have been lost. Moreover, two interchromosome telomereproximal crossings occurred to generate an end-end 


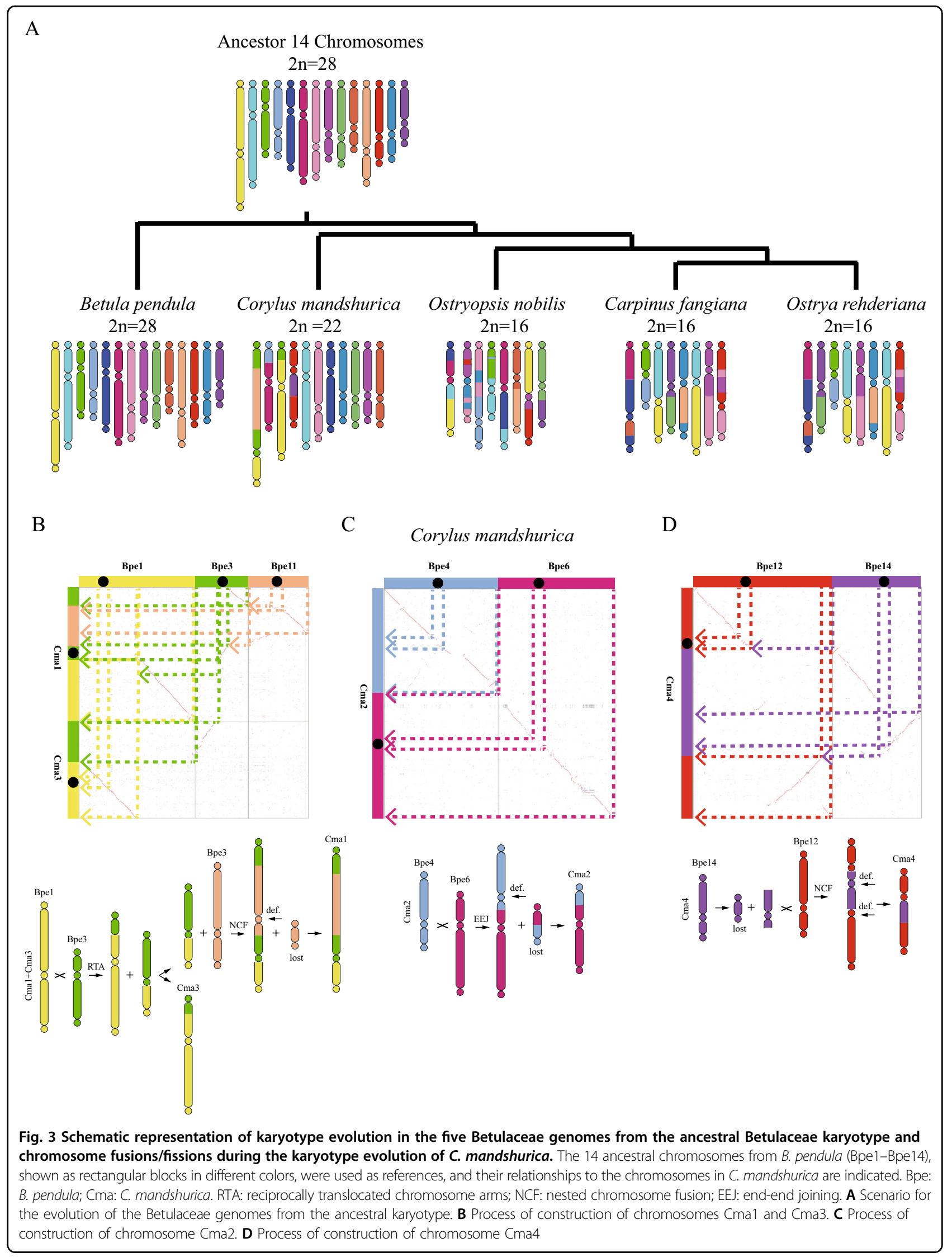


merged chromosome, two fusion chromosomes, and a satellite chromosome. The final chromosome number was reduced from 14 to 11 in $C$. mandshurica. Except for the genus Corylus, all the genera of Coryloideae (Carpinus, Ostryopsis, and Ostrya) had the same chromosome number $(n=8)$, and Ca. fangiana and Ostrya rehderiana also showed the same karyotype evolution trajectory (Supplementary Fig. 6), consistent with the predicted phylogenetic relationships. Both of them experienced two NCF, four EEJ, and four RTA events and lost six satellite chromosomes to produce the eight extant Ca. fangiana (Ostrya rehderiana) chromosomes (Supplementary Fig. 6). Ostryopsis nobilis exhibited the most complex evolutionary process, including seven EEJ and seven RTA events and the loss of five satellite chromosomes to form the extant karyotype (Supplementary Fig. 7).

To gain a further understanding of karyotype evolution in these Betulaceae species, we identified genes located within $10 \mathrm{~kb}$ around chromosome fission, fusion, or rearrangement events. A total of 19, 25, 33, and 39 genes were extracted in C. mandshurica, Ca. fangiana and Ostrya rehderiana and Ostryopsis nobilis, respectively. Among them, 2/1/1 genes related to chromosome structure were found in C. mandshurica, Ostrya rehderiana, Ostryopsis nobilis, respectively (Supplementary Table 14). We speculate that chromosome structure variations may cause genome instability, so related genes such as telomere protection and DNA repair are crucial. We did not detect genes that might be related to the traits that differ among Betulaceae species, such as kernel size, leaf properties, and adaptability. This may be because literature data on their functions are rare and further studies are needed, or it may be that the evolution of such traits is decided mainly by other mechanisms.

\section{Gene family evolution}

In C. mandshurica, 80 gene families comprising 290 genes exhibited significant rapid expansion relative to the ancestor of the hazels. Some of these families were annotated as phenylpropanoid metabolic process, aromatic compound catabolic process, regulation of salicylic acid-mediated signaling pathway, regulation of response to stress, and regulation of defense response (Supplementary Table 15). These genes may be a resource for investigating features specific to $C$. mandshurica.

We further identified R genes, which have an essential role in plant disease defense signaling ${ }^{15,16}$, in 12 species (Supplementary Table 16). A total of $80 \mathrm{R}$ genes containing the NBS domain were identified in the $C$. mandshurica genome; this is an intermediate number of NBS-containing genes (Supplementary Table 16 and Supplementary Fig. 8). Moreover, a cross-species comparison of numbers of $\mathrm{R}$ genes indicated that all Betulaceae species (49-116) except C. avellana (301) had far fewer than A. thaliana (178), Cas. equisetifolia (140), and J. regia (88). The higher number of $R$ genes within $C$. avellana maybe because the genome was obtained from an EFB-resistant breed (Jefferson) ${ }^{44,45}$. We also found larger gene numbers of two types of NBSs (CCNBS-LRR [CNL] and NBS-only) in both C. mandshurica and "Jefferson". CNL is one of the two major plant NBS proteins involved in pathogen recognition, and NBS-only genes may act as adaptors or regulators of CNL and TIRNBS-LRR proteins ${ }^{16}$. These genes may be important genetic resources for increasing disease resistance.

\section{Evolution of genes involved in oil biosynthesis}

Biosynthesis of oleic acid starts from acetyl-CoA and is catalyzed by multiple isozymes involved in 5 enzymatic steps, generating free oleic acid (Fig. 4). Then, long-chain acyl-CoA synthase (LACS) enzymes catalyze the synthesis of long-chain acyl-CoAs that feed into the Kennedy pathway, leading to the production of triacylglycerols ${ }^{46-49}$.

A total of 764 oil biosynthesis-related genes were identified in the C. mandshurica genome (Supplementary Table 17). Of these, 96 were identified as participating directly in oil biosynthesis (Fig. 4, Table 2, and Supplementary Fig. 9): including one acetyl-CoA carboxylase (ACCase) gene, five fatty acid biosynthase (FAB) genes, four $\beta$-ketoacyl-[acyl carrier protein] synthase II (KAS II) genes, three stearoyl-CoA desaturase (SAD) genes, seven fatty acyl-acyl carrier protein thioesterase A (FATA) genes, 33 long-chain acyl-CoA synthase (LACS) genes, 17 glycerol-3-phosphate acyltransferases (GPAT) genes, four lysophosphatidic acid acyl-transferase (LPAT) genes, 11 phosphatidate phosphatase (PAP) genes, four acyl-CoA: diacylglycerol acyl-transferase (DGAT) genes and seven phospholipid: diacylglycerol acyl-transferase (PDCT) genes. The number of fatty acid biosynthesis-related genes was within the range found in other plant species; there were 86 such genes in A. thaliana, 62 in B. pendula, 76 in C. avellana, 102 in Ca. fangiana, 96 in Cas. equisetifolia, 130 in J. regia, 81 in Ostrya rehderiana and 87 in Ostryopsis davidiana (Table 2). Of the proteins encoded by these genes, FATA and SAD are key enzymes in oleic acid biosynthesis (Fig. 4). There are 7 FATA and 3 SAD genes in the C. mandshurica genome, 4 FATA and $3 \mathrm{SAD}$ genes in the $A$. thaliana genome, and 10 FATA and 3 SAD genes in the walnut genome. Thus, the number of SAD genes in $C$. mandshurica is higher than that in $A$. thaliana but lower than that in walnut. The number of fatty acid biosynthesis-related genes in European hazelnut apparently exceeds only the number in $B$. pendula. The quality of the genome assembly may have affected this result, but FATA and SAD genes are still more numerous in European hazelnut than in $A$. thaliana.

\section{Rapidly evolving and positively selected genes}

To better reveal the evolutionary dynamics of C. mandshurica and identify more functional gene resources, we 


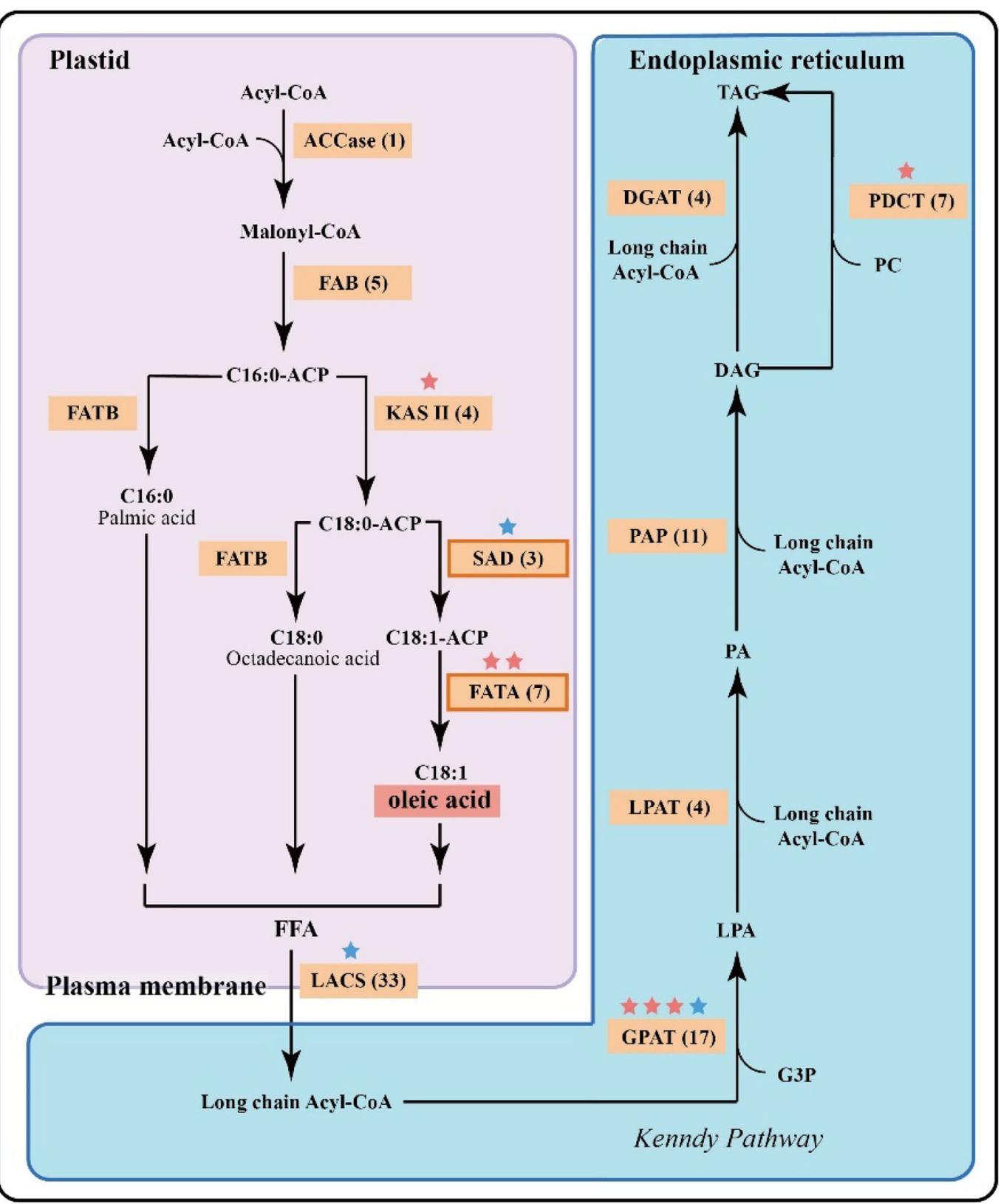

Fig. 4 Metabolic pathways and numbers of genes associated with biosynthesis and accumulation of oleic acid and TAG in C. mandshurica. Enzymes that participate in the de novo synthesis of free fatty acids and triacylglycerols (TAGs) are shown in rectangles, and key enzymes in oleic acid biosynthesis are indicated by boxes outlined in orange. The number of genes encoding each type of enzyme is shown in brackets. The red and blue stars indicate the enzymes under rapid evolution and positive selection, respectively. Acyl-CoA acyl-coenzyme A, DAG diacylglycerol, DGAT diacylglycerol acyl-transferase, FAB fatty acid biosynthesis, FATA fatty acyl-ACP thioesterase A, FATB fatty acyl-ACP thioesterase B, G3P glycerol-3 phosphate, GPAT glycerol-3-phosphate acyl-transferase, KAS II $\beta$-ketoacyl-[acyl carrier protein] synthase II, LACS long-chain acyl-CoA synthase, LPA lysophosphatidic acid, LPAT lysophosphatidyl acyl-transferase, PAP phosphatidic acid phosphatase, PA phosphatidic acid, PC phosphatidylcholine, PDCT phosphatidylcholine:diacylglycerol cholinephosphotransferase, SAD stearoyl ACP desaturase

further identified the rapidly evolving and positively selected genes within $C$. mandshurica by comparison with the other four Betulaceae species (B. pendula, Ca. fangiana, Ostryopsis nobilis, and Ostrya rehderiana) and Cas. equisetifolia. A total of 8624 high-confidence single-copy orthologs were obtained with a trimmed alignment length from 102 to $14,455 \mathrm{bp}$. The free-ratio model in PAML ${ }^{50}$ was first employed to estimate the independent $\mathrm{Ka} / \mathrm{Ks}$ ratio of each species based on all genes. We compared the overall whole-genome average Ka/Ks between six species 
Table 2 Cross-species comparison of the number of fatty acid biosynthesis-related gene families

\begin{tabular}{llllllllll}
\hline & Ath & Bpe & Cav & Ceq & Cfa & Cma & Jre & Ono & Ore \\
\hline ACCase & 2 & 1 & 1 & 3 & 2 & 1 & 2 & 1 & 1 \\
FAB & 6 & 3 & 4 & 9 & 12 & 5 & 8 & 9 & 3 \\
KAS II & 3 & 1 & 4 & 6 & 9 & 4 & 7 & 4 & 4 \\
SAD & 2 & 2 & 2 & 2 & 4 & 3 & 3 & 2 & 2 \\
FATA & 4 & 4 & 6 & 7 & 5 & 7 & 10 & 7 & 4 \\
LACS & 23 & 10 & 25 & 21 & 25 & 33 & 37 & 23 & 26 \\
GPAT & 20 & 18 & 15 & 20 & 18 & 17 & 30 & 18 & 18 \\
LPAT & 4 & 3 & 3 & 6 & 5 & 4 & 6 & 4 & 4 \\
PAP & 9 & 8 & 8 & 13 & 11 & 11 & 14 & 8 & 9 \\
DGAT & 7 & 3 & 2 & 1 & 4 & 4 & 4 & 3 & 3 \\
PDCT & 6 & 9 & 6 & 8 & 7 & 7 & 9 & 8 & 7 \\
Total & 86 & 62 & 76 & 96 & 102 & 96 & 130 & 87 & 81 \\
\hline
\end{tabular}

Ath A. thaliana, Bpe B. pendula, Cav C. avellana, Ceq Cas. equisetifolia, Cfa Ca. fangiana, Cma C. mandshurica, Fve F. vesca, Jre J. regia, Ono Ostryopsis nobilis, Ore Ostrya rehderiana, Acyl-CoA acyl coenzyme A, DAG diacylglycerol, DGAT diacylglycerol acyl-transferase, $F A B$ fatty acid biosynthesis, FATA fatty acyl-ACP thioesterase A, FATB fatty acyl-ACP thioesterase B, G3P glycerol-3 phosphate, GPAT glycerol-3-phosphate acyl-transferase, KAS /| $\beta$-ketoacyl-[acyl carrier protein] synthase II, LACS long-chain acyl-CoA synthase, LPA lysophosphatidic acid, LPAT lysophosphatidyl acyl-transferase, PAP phosphatidic acid phosphatase, $P A$ phosphatidic acid, $P C$ phosphatidylcholine, $P D C T$ phosphatidylcholine: diacylglycerol cholinephosphotransferase, SAD stearoyl ACP desaturase

and found that $C$. mandshurica showed a moderate evolutionary rate (Supplementary Fig. 10). In contrast, $B$. pendula has the highest $\mathrm{Ka} / \mathrm{Ks}$ ratio, possibly due to its short life history as a pioneer boreal tree ${ }^{25}$. We also identified 1327 rapidly evolving genes (REGs) and 1066 positively selected genes (PSGs) in C. mandshurica. Both types of genes were functionally enriched in organic compound metabolic activities, with 165 PSGs and 78 REGs involved in the heterocycle, carbohydrate, organic cyclic compound, and cellular aromatic compound metabolic processes (Supplementary Fig. 11). REGs further exhibit enrichment in glycerolipid biosynthetic and tetrapyrrole binding, which are associated with hazelnut flavor. Glycerolipids are the major nutrient component of hazelnut ${ }^{3-5}$, and organic cyclic compounds, especially pyrroles, are highly related to the volatile compounds of hazelnut $^{51}$. Within the oil biosynthesis pathway, three GPAT, SAD, and LACS genes were detected to be under positive selection, and three GPAT genes, two FATA genes, one KAS II gene, and one PDAT gene were found to be under rapid evolution (Supplementary Table 18). Both SAD and FATA, as the key enzymes in oleic acid biosynthesis $^{52}$, may underlie the high oleic acid concentration in hazelnuts. Moreover, within three significantly expanded transcription factor families that are involved in stress responses, i.e., the AP2/ERF-ERF, MYB, and CAMTA gene families, 11, 3, and 1 genes, respectively, were found to be positively selected; In the former two families, 15 and 6 genes, respectively, showed rapid evolution (Supplementary Table 18). Additionally, two R genes (RCNL type and NBS) were identified to be rapidly evolving (Supplementary Table 18). These rapidly evolving and positively selected genes may have contributed to the high environmental adaptability of C. mandshurica.

\section{Discussion}

The chromosome-level C. mandshurica genome assembly reported here has the highest quality among the recently published Betulaceae species genomes (Supplementary Table 3), with the longest contig N50 (14.8 Mb) and the highest genome completeness (97.2\%) in terms of BUSCO results. It could also be compared with the recently published genomes and at the top level (Supplementary Table 3). This high-quality genome may help us to identify biosynthetic pathways for oleic acid in hazelnuts, investigate the abiotic tolerance of this species and infer the evolutionary pathway of chromosomes of the hazelnut genus.

Hazelnut is one of the four major tree nuts consumed globally. Recent studies found that hazelnut oil is a good supplier of fatty acids, particularly oleic acid, which carries health benefits ${ }^{3,4}$. Although fatty acid synthesis mechanisms have been extensively studied in plants ${ }^{46-48}$, we identified candidate genes for the biosynthesis of oleic acid in hazel for the first time. These resources will be valuable in functional genomics studies and the improvement of traits that are economically important in hazelnut, such as nutrition and flavor. They included 96 genes whose products were identified as participating directly in the biosynthesis of oleic acid. Enzymes encoded by the latter group included ACCase, which is most likely the key enzyme determining the metabolic pathways that lead to oil or protein biosynthesis in the seed ${ }^{49,53}$. FATA and SAD are probably key components in oleic acid accumulation $^{54}$, and two FATA genes and one SAD gene showed rapid evolution and positive selection, respectively. Candidate genes related to fatty acid biosynthesis and undergoing rapid evolution or positive selection are highly valuable for genetic improvement of hazelnut in the future.

Stress resistance is among the most important traits in hazel breeding programs. C. mandshurica has a greater tolerance of fungal infection than cultivated forms of European hazel ${ }^{17}$. Overall, 80 genes with an NBS-coding region were identified in the $C$. mandshurica genome, whereas 301 were identified in European hazel, which has been bred for increased fruit output and disease resistance. We speculate that this is because the European hazel genome sequence was obtained from the EFBresistant diploid hazelnut accession "Jefferson", which 
may have retained a high number of NBS genes. Salicylic acid is a phytohormone that regulates signal transduction pathways involved in defense against biotic and abiotic stresses $^{54}$, and our evolutionary analysis found that several gene families related to salicylic acid metabolism and stress response had undergone rapid expansion in $C$. mandshurica. Moreover, three out of the four transcription factors in families that had significantly expanded in C. mandshurica were related to stress responses. Of these, MYB proteins are key factors in regulatory networks controlling development ${ }^{34}$, metabolism, and responses to biotic and abiotic stresses, AP2/ERF-ERFs have important functions in the transcriptional regulation of various responses to environmental stimuli $^{32}$, and CAMTA transcription factors are master regulators of salicylic acid-mediated immunity ${ }^{54}$. In addition, we identified several genes under rapid evolution or positive selection within these three TF families: MYB, AP2/ERF-ERF, and CAMTA. Taken together, these results indicate that NBS may not be the only key factor underlying the high-stress resistance of $C$. mandshurica; several other transcription factors may also contribute to this feature.

The rearrangement of centromeres and telomeres can result in changes in chromosome number ${ }^{55}$. The evolutionary trajectories of the Betulaceae chromosomes were reconstructed in this study. Previous studies have consistently agreed that the Betuloideae are more primitive than the Coryloideae in Betulaceae ${ }^{21,42,43}$, and indeed, $B$. pendula was located at the basal position in the phylogenetic relationship of these species. Thus, we considered that the B. pendula genome is the most primitive karyotype among the set of genomes currently available for Betulaceae species and used this genome as a reference. We used collinearity and phylogenetic analysis of the five Betulaceae species to provide evidence in support of an evolutionary scenario in which the most ancestral karyotype of the Betulaceae species investigated is that of $B$. pendula. Moreover, we found that the chromosomes of $B$. pendula are essentially preserved in the other four Betulaceae karyotypes, and in particular, a one-to-one correspondence between $B$. pendula and $C$. mandshurica further confirmed that $B$. pendula represents the ancestral karyotype. C. mandshurica occupies the second place because of the occurrence of only 2 NCFs, 1 chromosome end-end merge, 1 RTA, and the loss of 3 satellite chromosomes. Integration of phylogenomic and collinearity analyses of Betulaceae genomes further revealed that chromosomes evolved along three completely different trajectories within different genera of Coryloideae. Compared to Corylus, Ostryopsis-Carpinus-Ostrya showed a more sophisticated trajectory, and Carpinus and Ostrya had a consistent trajectory; all of these findings were in accordance with their phylogenetic relationships. With the availability of Alnus genome sequences, it will be possible to characterize the karyotype evolutionary trajectory for Betulaceae genomes in greater depth.

In summary, we obtained a high-quality chromosomelevel reference genome for the hazelnut species C. mandshurica and identified candidate genes related to oleic acid biosynthesis and stress tolerance in this species. Our genomic resources will guide hazelnut breeders in utilizing the excellent genetic resources of $C$. mandshurica and accelerate our understanding of genome evolution within the Betulaceae.

\section{Materials and methods \\ DNA extraction and sequencing}

Fresh young leaves were collected from a wild $C$. mandshurica tree growing in Xinglong Mountain National Nature Reserve in Lanzhou, Gansu Province, China ( $35^{\circ} 47^{\prime} 20.83^{\prime \prime} \mathrm{N}, 104^{\circ} 6^{\prime} 16.23^{\prime \prime}$ E, $\left.2290 \mathrm{~m}\right)$. Genomic DNA was extracted using a QIAGEN Genomic DNA extraction kit according to the standard operating procedure provided by the manufacturer. Quality control of the extracted DNA was carried out using a NanoDrop One UV-Vis spectrophotometer (ThermoFisher Scientific, USA) to check the DNA purity (OD260/280 ranging from 1.8 to 2.0 and OD260/230 between 2.0 and 2.2), and then a Qubit 3.0 A fluorometer (Invitrogen, USA) was used to accurately quantify the DNA. Since the sample was of adequate quality, a paired-end library with an insert size of $400 \mathrm{bp}$ was constructed using the standard Illumina protocol with the HiSeq X Ten platform. Following the Nanopore library construction protocol, a Nanopore library was constructed, and long-read data were generated using the PromethION sequencer platform (Oxford Nanopore Technologies, UK). Sequencing adapters were removed, and reads of low quality and short length were filtered out.

For Hi-C library construction, fresh young leaves from the same $C$. mandshurica tree were ground in liquid nitrogen. Chromatin was fixed using formaldehyde. Then, leaf cells were lysed, and the fixed chromatin was digested by the endonuclease DpnII. The $5^{\prime}$ overhangs of the DNA were recovered with biotin-labeled nucleotides, and the resulting blunt ends were ligated to each other using DNA ligase. Proteins were removed with protease to release the DNA molecules from the crosslinks. The purified DNA was sheared into 350-bp fragments and ligated to adaptors. The fragments labeled with biotin were extracted using streptavidin beads, and after PCR enrichment, the libraries were sequenced on the Illumina HiSeq X Ten platform.

\section{Genome assembly and pseudochromosome construction}

Before genome size estimation, we first filtered the short Illumina reads using fastp ${ }^{56}$ (v.0.20.0) with default parameters. Then, the k-mer ${ }^{57}$-based approach was selected to 
estimate the genome size. A total of $37.79 \mathrm{~Gb}$ clean reads were analyzed by Jellyfish ${ }^{58}$ (v.2.2.10) to generate the kmer depth distribution with a k-mer size of $21 \mathrm{bp}$, and GenomeScope $^{59}$ (v1.0.0) was used to estimate the genome size. Correction of Oxford Nanopore Technologies long reads and de novo assembly were performed by NextDenovo (v.2.1) with a seed cutoff of $19 \mathrm{~kb}$ and a read length cutoff of $1 \mathrm{~kb}$. After finishing the preassembly, iterative polishing was conducted using NextPolish ${ }^{23}$ (v.1.1). For genome polishing, Oxford Nanopore Technologies reads and Illumina sequencing reads were subjected to three rounds of genome correction. A subprogram of Purge Haplotigs ${ }^{60}$ was used to generate the final contig-level assembly to retain only one copy of each of the contigs from heterozygous regions. The completeness of the genome assembly was further assessed by $\mathrm{BUSCO}^{24}$ (v.3) with the Embryophyta_odb9 database.

For the Hi-C sequence data, we also initially filtered out low-quality reads by fastp ${ }^{56}$ (v.0.20.0) with default parameters. Then, HiCUP ${ }^{61}$ was applied to screen valid read pairs mapped uniquely to the primary assembly for further analysis. ALLHiC ${ }^{62}$ (v0.8.12) was used in simple diploid mode to scaffold the genome and optimize the ordering and orientation of each clustered group, producing a chromosomal level assembly, and finally, a heatmap was plotted.

\section{Repeat element identification}

RepeatMasker ${ }^{63}$ and RepeatProteinMasker ${ }^{63}$ were used to identify repetitive elements based on homology alignments between $C$. mandshurica genome sequences and Repbase (v.16.10). We then applied the de novo approach to improve the sensitivity of our repeat identification. Briefly, RepeatModeler ${ }^{64}$ and LTR_Finder ${ }^{65}$ (v1.06) were selected to construct the repeat library, and then RepeatMasker ${ }^{63}$ was employed to generate the de novo predictions.

\section{Gene prediction}

A combination of transcriptome-based, homologybased, and de novo approaches was used to accurately predict high-quality protein-coding genes. To predict genes ab initio, Augustus ${ }^{66}$ (v.3.2.3), GenScan, and GlimmerHMM $^{67}$ (v.3.0.4) were employed using a model trained based on coding sequence (CDS) data from $A$. thaliana. For homology-based prediction, protein sequences from A. thaliana, C. avellana, Ca. fangiana, Cas. equisetifolia, Carica papaya, F. vesca, Ostryopsis nobilis, $P$. persica, and $Q$. robur were used. For transcriptome-based prediction, the nonredundant fulllength transcripts from the de novo assembly were aligned to the genome to resolve gene structures using PASA. EVidenceModeler $^{68}$ (EVM, v.1.1.1) was used to generate the final consensus set of gene models obtained using the three approaches. Functional annotation of proteincoding genes was performed by BLASTP ${ }^{69}$ (v.2.7.1+) (Evalue $<1 \times 10^{-5}$ ) using SwissProt ${ }^{30}$ and TrEMBL $^{30}$. InterProScan ${ }^{70}$ (v.5.28) and $\mathrm{Hmmer}^{71}$ (v3.1b2) were used to annotate protein domains by searching the InterPro and Pfam databases, respectively. Gene Ontology (GO) terms for each gene were obtained from the corresponding InterPro or Pfam entry. The pathways in which each gene might be involved were assigned by BLAST against the Kyoto Encyclopedia of Genes and Genomes ${ }^{30}$ (KEGG) database. Moreover, the transcription factors in C. mandshurica and C. avellana were detected using iTAK $^{72}$ and PlantTFDB ${ }^{73}$. To test the significance of gene number variation among different species, the $2 * 2$ contingency table $\chi^{2}$ test was executed for each transcription factor gene family based on the family gene number and the species total gene number.

\section{Genome evolution and expansion/contraction of gene families}

To investigate the evolutionary trajectory of the $C$. mandshurica genome, a total of 11 other species were selected for phylogenetic analysis: A. thaliana, B. pendula, C. avellana, Ca. fangiana, Cas. equisetifolia, F. vesca, J. regia, Ostrya rehderiana, Ostryopsis nobilis (unpublished genomic data), Q. robur and V. vinifera. An all-vs-all BLASTP $^{69}$ (v2.2.26) (E-value cutoff: $1 \times 10^{-5}$ ) was first employed to generate similarity information for all genes. Then, we identified high-quality single-copy genes by applying OrthoMCL ${ }^{74}$ and constructed a phylogenetic tree with this gene set using $\mathrm{RAxML}^{75}$ (v8.0.0). We further estimated the times of divergence between species with $\mathrm{MCMCtree}^{76}$ in the PAML ${ }^{49}$ package (v4). The divergence time between $A$. thaliana and $V$. vinifera (107-135 Mya) acquired from TimeTree (http://www. timetree.org/) was used as the calibration point. Gene family expansion and contraction were further estimated by $\operatorname{CAFE}^{77}$ (v4.2) using the gene cluster information and the estimated time tree. The parameter $\lambda$ was estimated along each branch with the random model, and then all the gene families were classified into three types: expanded, contracted, or unchanged.

\section{Evolution genes identified}

We selected six genomes, i.e., those of B. pendula, $C a$. fangiana, C. mandshurica, Ostryopsis nobilis, and Ostrya rehderiana to identify orthologs for analyzing positive selection and used Cas. equisetifolia as outgroup. First, Sonicparanoid ${ }^{78}$ was used to detect orthologs among the six genomes. Next, we used the PAML ${ }^{49} \mathrm{v} 4.8$ pipeline for genome-wide detection of the genes with positive selection or rapid evolution specified in the $C$. mandshurica clade as the foreground branch. Finally, PSGs and REGs were identified based on an adjusted $P$-value $<0.05$. 


\section{Synteny and dot plot generation for the analysis of chromosome evolution}

For genomes with pseudochromosomes, syntenic blocks between pairs of genomes, defined as a region containing more than five collinear genes, were searched for by the MCScanX ${ }^{79}$ package. The results were represented visually in combination with the phylogenetic tree. Ks of the collinear orthologous gene pairs was determined using the Perl script "add_ka_and_ks_to_collinearity.pl" implemented in MCScanX $\mathrm{X}^{79}$. CDS anchors between every possible pair of chromosomes in multiple genomes were searched for using BLASTP ${ }^{69}\left(E\right.$-value $\left.<1 \times 10^{-5}\right)$. The best matches were displayed in red, and other matches were displayed in blue to help distinguish orthology from paralogy. All dot plots were drawn by WGDI (https://pypi. org/project/WGDI/).

\section{Acknowledgements}

This work was supported by the Strategic Priority Research Program of the Chinese Academy of Sciences (XDB31010300), the National Key Research and Development Program of China (2017YFC0505203), and the National Natural Science Foundation of China (31590821 and 31900201). All the computation works were supported by Supercomputing Center of Lanzhou University and Big Data Computing Platform for Western Ecological Environment and Regional Development.

\section{Author details}

${ }^{1}$ State Key Laboratory of Grassland Agro-Ecosystem, Institute of Innovation Ecology \& School of Life Sciences, Lanzhou University, Lanzhou, China. ${ }^{2}$ Key Laboratory of Bio-Resource and Eco-Environment of Ministry of Education \& State Key Laboratory of Hydraulics \& Mountain River Engineering, College of Life Sciences, Sichuan University, Chengdu, China. ${ }^{3}$ CAS Key Laboratory of Tropical Forest Ecology, Xishuangbanna Tropical Botanical Garden, Chinese Academy of Sciences, 666303 Mengla, Yunnan, China. ${ }^{4}$ Center of Plant Ecology, Core Botanical Gardens, Chinese Academy of Sciences, 666303 Mengla, Yunnan, China

\section{Author contributions}

Y.Y. and J.L. conceived and designed the project. J.C., Z.L., Y.L., and H.H. collected samples for DNA and RNA extraction. Y.Y. and J.L. supervised the bioinformatics analysis. Y.L. performed the genome assembly and annotation. Z.W. and J.Y. performed chromosome-scale assembly. P.S. performed karyotype evolution analysis. P.S., Y.L., Y.W., and J.M. performed the gene family evolution analysis. X.D. and Z.Z. performed phylogenetic analysis. Y.Y., J.L., and Y.L. wrote the draft manuscript. All authors read and approved the final version of the manuscript.

\section{Data availability}

All the raw sequence reads (including the Nanopore long reads, NGS short reads and $\mathrm{Hi}-\mathrm{C}$ reads) used in this study have been deposited at $\mathrm{NCBI}$ under the BioProject accession number PRJNA638027. The genome assembly file and genome annotation files (repeat annotation and gene structure annotation) are available at figshare (doi.org/10.6084/m9.figshare.12523124.v1).

\section{Conflict of interest}

The authors declare no competing interests.

Supplementary information The online version contains supplementary material available at https://doi.org/10.1038/s41438-021-00495-1.

Received: 22 June 2020 Revised: 11 January 2021 Accepted: 20 January 2021

Published online: 01 March 2021

\section{References}

1. Wu, Z. Vegetation in China (1995).

2. Wang, G. Progress in cultivation and utilization of Corylus L. Resources in China (I) - Corylus germplasm resources. For. Res. 31, 105-112 (2018).

3. Ji, J. M., Ge, Z. F., Feng, Y. S. \& Wang, X. D. Lipid characterization of Chinese Wild Hazelnuts (Corylus mandshurica Maxim.). J. Oleo Sci. 68, 13-20 (2019).

4. Alasalvar, C., Amaral, J. S. \& Shahidi, F. Functional lipid characteristics of Turkish Tombul hazelnut (Corylus avellana L.). J. Agr. Food Chem. 54, 10177-10183 (2006).

5. Tufekci, F. \& Karatas, S. Determination of geographical origin Turkish hazelnuts according to fatty acid composition. Food Sci. Nutr. 6, 557-562 (2018).

6. Teres, S. et al. Oleic acid content is responsible for the reduction in blood pressure induced by olive oil. Proc. Natl Acad. Sci. USA 105, 13811-13816 (2008).

7. Cerain, L. D. \& Adela. in Mutagenic Act. Meat Samples Deep-Fry Olive Oil Vol. 106, 989-996 (2010).

8. Perdomo, L. et al. Protective role of oleic acid against cardiovascular insulin resistance and in the early and late cellular atherosclerotic process. Cardiovasc. Diabetol. 14, 75 (2015).

9. Boccacci, P. \& Botta, R. Investigating the origin of hazelnut (Corylus avellana L.) cultivars using chloroplast microsatellites. Genet. Resour. Crop Evol. 56, 851-859 (2009).

10. Johnson, K. B. et al. Eastern filbert blight of European hazelnut: It's becoming a manageable disease. Plant Dis. 80, 1308-1316 (1996).

11. Bhattarai, G., Mehlenbacher, S. \& Smith, D. C. Novel sources of resistance to Eastern Filbert blight in Hazelnut. Hortscience 50, S401-S401 (2015).

12. Kask, K. Nut quality of wild European hazelnut in Estonia and attempts at hazelnut breeding. Acta Hortic. 556, 37-40 (2001).

13. Li, T. D. et al. Domestication of wild tomato is accelerated by genome editing. Nat. Biotechnol. 36, 1160-1165 (2018).

14. Chen, Y. Z. et al. High oleic acid content, nontransgenic allotetraploid cotton (Gossypium hirsutum L.) generated by knockout of GhFAD2 genes with CRISPR/Cas9 system. Plant Biotechnol. J. (2020).

15. Dangl, J. L. \& Jones, J. D. G. Plant pathogens and integrated defence responses to infection. Nature 411, 826-833 (2001).

16. Meyers, B. C. et al. Genome-wide analysis of NBS-LRR-encoding genes in Arabidopsis. Plant Cell 15, 809-834 (2003).

17. Clarice, J. C., Shawn, A. M. \& David, C. S. Sources of resistance to Eastern Filbert Blight in Hazelnut. J. Am. Soc. Hortic. Sci. 123, 253-257 (1998).

18. Erdogan, V. \& Mehlenbacher, S. A. Interspecific hybridization in hazelnut (Corylus). J. Am. Soc. Hortic. Sci. 125, 489-497 (2000).

19. Robert, H. W. Cytological studies on the Betulaceae. II. Coryolus Alnus.Chic. J. 88, 383-399 (1929).

20. Robert, H. W. Cytological studies on the Betulaceae. IV. Betula, Carpinus, Ostrya, Ostryopsis. Chic. J. 90, 108-115 (1930).

21. Chen, Z. Phylogeny ang phytogeography of the Betulaceae. Acta Phyytotax. Sin. 32, 1-31 (1994)

22. Rowley, E. R. et al. A draft genome and high-density genetic map of European Hazelnut (Corylus avellana L.). Preprint at https://www.biorxiv.org/content/ 10.1101/469015V1 (2018)

23. Hu, J., Fan, J. P., Sun, Z. Y. \& Liu, S. L. NextPolish: a fast and efficient genome polishing tool for long-read assembly. Bioinformatics 36, 2253-2255 (2020).

24. Simao, F. A. et al. BUSCO: assessing genome assembly and annotation completeness with single-copy orthologs. Bioinformatics 31, 3210-3212 (2015).

25. Salojarvi, J. et al. Genome sequencing and population genomic analyses provide insights into the adaptive landscape of silver birch. Nat. Genet. 49, 904-915 (2017).

26. Yang, X. Y. et al. A chromosome-level reference genome of the hornbeam, Carpinus fangiana. Sci. Data 7, 1-24 (2020).

27. Yang, Y. Z. et al. Genomic effects of population collapse in a critically endangered ironwood tree Ostrya rehderiana. Nat. Commun. 9, 5449-5457 (2018).

28. Wang, Z. F. et al. Hybrid speciation via inheritance of alternate alleles of parental isolating genes. Mol. Plant 14, 208-222 (2020).

29. Chen, F. et al. The sequenced angiosperm genomes and genome databases. Front. Plant Sci. 9, 418 (2018).

30. Bairoch, A. \& Apweiler, R. The SWISS-PROT protein sequence database and its supplement TrEMBL in 2000. Nucleic Acids Res. 28, 45-48 (2000).

31. Kanehisa, M. \& Goto, S. KEGG: Kyoto encyclopedia of genes and genomes. Nucleic Acids Res 28, 27-30 (2000). 
32. Xu, Z. S., Chen, M., Li, L. C. \& Ma, Y. Z. Functions and application of the AP2/ERF transcription factor family in crop improvement. J. Integr. Plant Biol. $\mathbf{5 3}$ 570-585 (2011).

33. Doherty, C. J., Van Buskirk, H. A., Myers, S. J. \& Thomashow, M. F. Roles for Arabidopsis CAMTA transcription factors in cold-regulated gene expression and freezing tolerance. Plant Cell 21, 972-984 (2009).

34. Roy, S. Function of MYB domain transcription factors in abiotic stress and epigenetic control of stress response in plant genome. Plant Signal Behav. 11, 1-7 (2016).

35. Toledo-Ortiz, G., Huq, E. \& Quail, P. H. The Arabidopsis basic/helix-loop-helix transcription factor family. Plant Cell 15, 1749-1770 (2003).

36. Ye, G. F. et al. De novo genome assembly of the stress tolerant forest species Casuarina equisetifolia provides insight into secondary growth. Plant J. 97 779-794 (2019).

37. Martinez-Garcia, P. J. et al. The walnut (Juglans regia) genome sequence reveals diversity in genes coding for the biosynthesis of non-structural polyphenols. Plant J. 87, 507-532 (2016).

38. Plomion, $\mathrm{C}$. et al. Oak genome reveals facets of long lifespan. Nat. Plants $\mathbf{4}$, 440-452 (2018)

39. Zapata, L. et al. Chromosome-level assembly of Arabidopsis thaliana Ler reveals the extent of translocation and inversion polymorphisms. Proc. Natl Acad. Sci. USA 113, E4052-E4060 (2016).

40. Buti, M. et al. The genome sequence and transcriptome of Potentilla micrantha and their comparison to Fragaria vesca (the woodland strawberry). Gigascience 7, 1-41 (2018).

41. Jaillon, $\mathrm{O}$. et al. The grapevine genome sequence suggests ancestral hexaploidization in major angiosperm phyla. Nature 449, 463-465 (2007).

42. Chen, Z. D., Manchester, S. R. \& Sun, H. Y. Phylogeny and evolution of the Betulaceae as inferred from DNA sequences, morphology, and paleobotany. Am. J. Bot. 86, 1168-1181 (1999).

43. Chen, Z. D. \& Lu, A. M. Phylogeny and evolution od Betulaceae. China Acad. J. 3, 189-191 (2001)

44. Kosztarab, M., Roane, M. K. \& Drake, C. R. Reduction of Eastern Filbert Blight on Corylus Avellana. Phytopathology 70, 690-690 (1980).

45. Sathuvalli, V., Mehlenbacher, S. A. \& Smith, D. C. High-resolution genetic and physical mapping of the eastern filbert blight resistance region in 'Jefferson' Hazelnut (Corylus avellana L.). Plant Genome 10, 1-12 (2017).

46. Bates, P. D., Stymne, S. \& Ohlrogge, J. Biochemical pathways in seed oil synthesis. Curr. Opin. Plant Biol. 16, 358-364 (2013)

47. Thelen, J. J. \& Ohlrogge, J. B. Metabolic engineering of fatty acid biosynthesis in plants. Metab. Eng. 4, 12-21 (2002)

48. Ohlrogge, J. B. Design of new plant-products - engineering of fatty-acid metabolism. Plant Physiol. 104, 821-826 (1994).

49. Nikolau, B. J., Ohlrogge, J. B. \& Wurtele, E. S. Plant biotin-containing carboxylases. Arch. Biochem. Biophys. 414, 211-222 (2003).

50. Yang, Z. H. PAML: a program package for phylogenetic analysis by maximum likelihood. Comput. Appl. Biosci. 13, 555-556 (1997).

51. Alasalvar, C., Shahidi, F. \& Cadwallader, K. R. Comparison of natural and roasted turkish tombul hazelnut (corylus avellana l.) volatiles and flavor by $\mathrm{dha} / \mathrm{gc} / \mathrm{ms}$ and descriptive sensory analysis. J. Agric. Food Chem. 51, 5067-5072 (2003).

52. Dormann, P., Voelker, T. A. \& Ohlrogge, J. B. Cloning and expression in Escherichia coli of a novel Thioesterase from Arabidopsis thaliana specific for long-chain acyl-acyl carrier proteins. Arch. Biochem. Biophys. 316, 612-618 (1995).

53. Roesler, K. et al. Targeting of the Arabidopsis homomeric acetyl-coenzyme A carboxylase to plastids of rapeseeds. Plant Physiol. 113, 75-81 (1997).

54. Kim, Y. S. et al. CAMTA-mediated regulation of salicylic acid immunity pathway genes in Arabidopsis exposed to low temperature and pathogen infection. Plant Cell 29, 2465-2477 (2017).
55. Wang, Z. Y. \& Wang, X. Y. Evolutionary genomics model chromosome number reduction B chromosome production. Sci. Sin. Vitae 50, 524-537 (2020).

56. Chen, S. F., Zhou, Y. Q., Chen, Y. R. \& Gu, J. fastp: an ultra-fast all-in-one FASTQ preprocessor. Bioinformatics 34, 884-890 (2018).

57. Li, R. Q. et al. The sequence and de novo assembly of the giant panda genome. Nature 463, 311-317 (2010).

58. Marcais, G. \& Kingsford, C. A fast, lock-free approach for efficient parallel counting of occurrences of k-mers. Bioinformatics 27, 764-770 (2011).

59. Vurture, G. W. et al. GenomeScope: fast reference-free genome profiling from short reads. Bioinformatics 33, 2202-2204 (2017).

60. Roach, M. J., Schmidt, S. A. \& Borneman, A. R. Purge Haplotigs: allelic contig reassignment for third-gen diploid genome assemblies. BMC Bioinformatics 19, 460 (2018).

61. Connell, L. W., Sexton, F. W. \& Prinja, A. K. Further development of the heavy ion cross section for single event UPset: model (HICUP). IEEE Trans. Nucl. Sci. 42, 2026-2034 (1995).

62. Zhang, X. T. et al. Assembly of allele-aware, chromosomal-scale autopolyploid genomes based on Hi-C data. Nat. Plants 5, 833-845 (2019).

63. Chen, N. in Using RepeatMasker to Identify Repetitive Elements in Genomic Sequences Ch. 4 (ed. Andreas, D. B.) (2004).

64. Bao, W. D., Kojima, K. K. \& Kohany, O. Repbase Update, a database of repetitive elements in eukaryotic genomes. Mob. DNA-Uk 6, 1-6 (2015).

65. Xu, Z. \& Wang, H. LTR_FINDER: an efficient tool for the prediction of full-length LTR retrotransposons. Nucleic Acids Res. 35, W265-W268 (2007).

66. tanke, M. \& Morgenstern, B. AUGUSTUS: a web server for gene prediction in eukaryotes that allows user-defined constraints. Nucleic Acids Res. 33, W465-W467 (2005).

67. ajoros, W. H., Pertea, M. \& Salzberg, S. L. TigrScan and GlimmerHMM: two open source ab initio eukaryotic gene-finders. Bioinformatics 20, 2878-2879 (2004).

68. Haas, B. J. et al. Automated eukaryotic gene structure annotation using EVidenceModeler and the program to assemble spliced alignments. Genome Biol. 9, R7 (2008).

69. Altschul, S. F. et al. Gapped BLAST and PSI-BLAST: a new generation of protein database search programs. Nucleic Acids Res. 25, 3389-3402 (1997).

70. Zdobnov, E. M. \& Apweiler, R. InterProScan—an integration platform for the signature-recognition methods in InterPro. Bioinformatics 17, 847-848 (2001).

71. Reagan, R. L. \& Bernstein, R. L. Data mining of signaling proteins using the HMMER method: high selectivity for protein sequence homology searches. Proc. Int. Conf. Math. Eng. Tech. Med. Biol. Sci. sl, II, 185-191 (2000).

72. Zheng, Y. et al. iTAK: a program for genome-wide prediction and classification of plant transcription factors, transcriptional regulators, and protein kinases. Mol. Plant 9, 1667-1670 (2016).

73. Tian, F. et al. PlantRegMap: charting functional regulatory maps in plants. Nucleic Acids Res. 48, D1104-D1113 (2020).

74. Li, L., Stoeckert, C. J. \& Roos, D. S. OrthoMCL: Identification of ortholog groups for eukaryotic genomes. Genome Res. 13, 2178-2189 (2003).

75. Stamatakis, A. RAxML version 8: a tool for phylogenetic analysis and postanalysis of large phylogenies. Bioinformatics 30, 1312-1313 (2014).

76. Puttick, M. N. MCMCtreeR: functions to prepare MCMCtree analyses and visualize posterior ages on trees. Bioinformatics 35, 5321-5322 (2019).

77. De Bie, T., Cristianini, N., Demuth, J. P. \& Hahn, M. W. CAFE: a computational tool for the study of gene family evolution. Bioinformatics 22, 1269-1271 (2006).

78. Salvatore, C. \& Wataru, I. Sonicparanoid: fast, accurate, and easy orthology inference. Bioinformatics 1, 1-3 (2018).

79. Wang, Y. P. et al. MCScanX: a toolkit for detection and evolutionary analysis of gene synteny and collinearity. Nucleic Acids Res. 40, 1-14 (2012). 